\title{
FLEXIBILIDADE EM IDOSAS PRATICANTES DE GINÁSTICA FUNCIONAL
}

\author{
Zenite Machado ${ }^{1}$ \\ Pamela Boing dos Santos ${ }^{2}$ \\ Adriana Coutinho de Azevedo Guimarães ${ }^{3}$ \\ Simone Korn ${ }^{4}$ \\ Melissa de Carvalho Souza ${ }^{5}$
}

resumo

Objetivou-se analisar a flexibilidade em idosas praticantes de ginástica funcional. O estudo causal comparativo, com uma amostra de 50 idosas, coletou os dados pelo protocolo de Rikli e Jones. Foram comparados os valores médios intragrupos, entre o pré e pós-teste, entre os grupos etários e os percentuais em cada categoria. Os testes

1 Graduada em Educação Física. Doutora em Ciências da Motricidade Humana. Professora Adjunta da Universidade do Estado de Santa Catarina (UDESC), vinculada ao Departamento de Educação Física. E-mail: zenite13@yahoo.com.br.

2 Graduada em Educação Física. E-mail: pamelaboing@hotmail.com.

3 Graduada em Educação Física. Doutora em Ciências da Motricidade Humana. Professora Adjunta da Universidade do Estado de Santa Catarina (UDESC), vinculada ao Departamento de Educação Física. E-mail: nanaguim@terra.com.br.

4 Graduada em Educação Física. Especialização em Obesidade e Emagrecimento.

E-mail: simonekorn@gmail.com.

5 Graduada em Educação Física. Mestre em Ciências do Movimento Humano.

E-mail: mecarvalho.s@gmail.com. 
estatísticos realizados foram respectivamente: teste "t" de Student pareado e não pareado, ANOVA e quil-quadrado de Pearson $(p \leq 0,05)$. O resultado foi significativo na flexibilidade dos membros inferiores e membros superiores entre os grupos. Portanto, um programa de intervenção de ginástica funcional colabora na melhoria e manutenção da flexibilidade em idosas.

palavras - chave

Envelhecimento. Atividade Motora. Elasticidade.

\section{Introdução}

O envelhecimento é o grande desafio a ser enfrentado por todos os países atualmente, dos desenvolvidos até os emergentes. De acordo com o Instituto Brasileiro de Geografia e Estatística, no Brasil, a população idosa era de 8,6 \% da população total em 2000, o que até 2020 será de aproximadamente 31,8 milhões de pessoas, cerca de $13 \%$ da população brasileira. A população idosa feminina é mais numerosa. Em 1991, elas correspondiam a 54\% da população de idosos e, em 2000 , foi verificado um aumento para $55,1 \%$, significando aproximadamente 100 mulheres para 81,6 homens, o que em 1991 era de 100 mulheres para 85,2 homens. Esse diferencial de expectativa de vida pode ser em decorrência do cuidado diferenciado com a saúde e uma maior adesão a programas de atividade física por parte das mulheres (CARVALHO; PETREÇA, 2012).

Com o aumento da população idosa no Brasil e no mundo, há uma maior incidência de doenças crônico-degenerativa, bem como de incapacidades decorrentes desse processo (VERAS, 2009). Ainda, ocorre nesse período um declínio da condição funcional do indivíduo, que embora seja decorrente do envelhecimento, se não tratado, pode acarretar em limitações mais severas (VIRTUOSO JÚNIOR; GUERRA, 2008).

As limitações funcionais em idosos ocorrem por diversos fatores, sendo o mais preocupante deles o sedentarismo. No Brasil, o sistema de vigilância de fatores de risco e proteção para doenças crônicas não transmissíveis por inquérito telefone (VIGITEL) pesquisou 54.369 indivíduos de ambos os sexos, com idade igual ou superior a 18 anos e verificou que a frequência de condição de sedentarismo foi máxima na faixa etária acima dos 65 anos, ficando em $56,1 \%$, sendo $65,4 \%$ para os homens e 50,3\% para as mulheres (MAZO et al., 2008). 
Para uma efetiva prevenção, ou mesmo para a redução das perdas funcionais associadas ao envelhecimento, sugere-se a inclusão de idosos em programas regulares de exercícios físicos (BENEDETTI; GONÇALVES; MOTA, 2007), os quais, por sua vez, podem, ainda, minimizar o declínio do desempenho físico e contribuir para melhoras significativas na força do idoso que o pratica (FREITAS et al., 2007). Ao mesmo tempo, auxiliam na manutenção da capacidade física, aumentando a qualidade de vida, diminuindo o risco de quedas e mantendo ou melhorando a sua funcionalidade (SILVA et al., 2010).

Dentre todas as atividades físicas indicadas para idosos, destaca-se o trabalho da flexibilidade, capacidade física responsável pela execução angular máxima de uma articulação ou de um conjunto de articulações, dentro dos limites morfológicos, sem o risco de provocar lesões, sendo que uma pessoa bem condicionada fisicamente consegue manter sua flexibilidade em níveis satisfatórios (ACHOUR JUNIOR, 2009). Bons níveis de flexibilidade em idosos proporcionam benefícios para o desempenho motor, sendo importante na execução de movimentos simples do dia a dia ou para atividades complexas pelas quais possam vir a realizar, bem como para a preservação da saúde (SILVA; RABELO, 2006).

Segundo Rikli e Jones (2008), a flexibilidade é importante para a execução de tarefas comuns do cotidiano e mobilidade do idoso como pentear o cabelo, fechar o zíper de uma roupa, subir degraus, entrar ou sair de um carro. Além disso, contribui para a prevenção de lombalgias e lesões musculoesqueléticas, e ainda na redução significativa do risco de quedas em idosos (BENEDETTI et al., 2008; GALLON; GOMES, 2011; OH et al., 2012). A redução da amplitude de movimentação da cintura escapular pode resultar em aumento de chance de lesão, incapacidade física, dor e instabilidade postural (RIKLI; JONES, 2008).

A literatura tem apontado para a importância de uma boa flexibilidade para o desenvolvimento do idoso, sendo que falta de uma boa flexibilidade pode ocasionar, entre muitos problemas, dor e limitações de movimentos (ALVES et al., 2004; CIPRIANI et al., 2010). Dessa forma, surgiu o interesse pela realização de um estudo com o objetivo de analisar a treinabilidade da flexibilidade em idosas de Florianópolis.

\section{Métodos}

O estudo foi aprovado pelo Comitê de Ética em Pesquisa em Seres Humanos da Universidade do Estado de Santa Catarina (UDESC), protocolo n. 63431 de 30/07/2012. Como a amostra foi proveniente de dois grupos de 
idosas que praticam ginástica funcional oferecida pelo programa Viver Ativo, foi solicitada à coordenação desse programa acesso ao banco de dados das avaliações realizadas nos meses de março e novembro de 2011.

Este estudo caracteriza-se como causal comparativo, por considerar as variáveis sexo e idade, e descritivo. O programa Viver Ativo, hoje em dia, oferece a modalidade de ginástica funcional a 15 grupos de ginástica, três vezes por semana, sendo a duração da sessão de 60 minutos. As sessões são ministradas por acadêmicos do curso de Bacharelado em Educação Física a partir da quinta fase. Na concepção do programa Viver Ativo, a ginástica funcional é uma modalidade de prática que envolve a realização de exercícios de força e resistência dos membros, flexibilidade, agilidade e resistência aeróbica, para a qual é utilizado apenas o próprio corpo, dispensando implementos.

A amostra, intencional, foi composta por 50 idosas participantes de dois grupos de ginástica (Canto do Rio e Capoeiras). Esses grupos foram os selecionados por serem aqueles com maior índice de aderência (grupos com baixa rotatividade e com idosas com tempo de prática superior a um ano). O programa conta com avaliações periódicas que ocorrem duas vezes ao ano (no início das atividades e no final do ano antes das férias), em março e novembro, respectivamente.

Como critérios de inclusão foram adotados a idade (mínima de 60 e máxima de 80 anos) e o sexo (somente o feminino). A idade mínima de 60 anos justifica-se pelo fato de que é a que permite a categorização de um indivíduo como idoso. O estudo exclusivamente com mulheres justifica-se pelo fato de que os homens participam muito pouco desse tipo de atividade, e o número reduzido de sujeitos dificulta a utilização de testes estatísticos inferenciais, principalmente se for considerado que o protocolo que será utilizado ainda estratifica os sujeitos por faixas etárias. Os critérios de exclusão foram idade inferior a 60 anos e superior a 80 e a não participação inferior a um ano no programa Viver Ativo.

O instrumento utilizado para coleta de dados foi o teste desenvolvido por Rikli e Jones (2008), sendo utilizadas apenas as variáveis relacionadas à flexibilidade dos membros superiores e inferiores. Para avaliar a flexibilidade dos membros inferiores, utiliza-se uma cadeira com encosto e sem braços com o assento a uma altura de aproximadamente $43 \mathrm{~cm}$, e uma régua de $45 \mathrm{~cm}$. $\mathrm{O}$ avaliado senta-se na cadeira mantendo uma perna a flexionada e o pé do chão, e a perna preferida estendida à frente com o calcanhar no cão. $\mathrm{O}$ avaliado tenta tocar os dedos dos pés escorregando as mãos, uma em cima da outra, com as pontas dos dedos médios, na perna estendida, mantendo a posição por dois segundos. Com uma régua, o avaliador registra a distância $(\mathrm{cm})$ até os 
dedos dos pés (resultado mínimo) ou a distância ( $\mathrm{cm}$ ) que o avaliado consegue alcançar para além dos dedos dos pés (resultado máximo). O meio do dedo grande do pé representa o ponto zero. O melhor resultado em duas tentativas é usado para avaliar o desempenho.

A avaliação da flexibilidade dos membros superiores (ombro) é realizada com o avaliado de pé, estando o avaliador atrás do avaliado. O avaliado coloca a mão preferida sobre o mesmo ombro, a palma aberta e os dedos estendidos, alcançando o meio das costas tanto quanto possível (cúbito apontado para cima). A mão do outro braço deverá ser colocada atrás das costas, a palma para cima, alcançando para cima o mais distante possível na tentativa de tocar ou sobrepor os dedos médios estendidos de ambas as mãos. Sem mover as mãos de avaliado, o avaliador ajuda a verificar se os dedos médios de cada mão estão direcionados um ao outro. Os resultados negativos (-) representam a distância mais curta entre os dedos médios; os resultados positivos $(+)$ representam a medida da sobreposição dos dedos médios. Registram-se as duas medidas. $\mathrm{O}$ "melhor" valor é usado para medir o desempenho.

Todos os testes foram aplicados nas mesmas condições em todos os períodos das duas coletas. O protocolo estabelece uma mesma classificação para todas as variáveis estudadas, e os resultados são separados por percentis e classificados como: abaixo da média da população (1), na média da população (2), e acima da média (3), para essa classificação dos dados.

Para a categorização, os resultados são separados por gênero e por grupo de idade, divididos em cinco categorias etárias: a) 60 a 64 anos, b) 65 a 69 anos, c) de 70 a 74, d) 75 ou mais.

A partir do acesso ao banco de dados, foram captados os resultados: idade, estatura, massa corporal e os resultados dos testes de flexibilidade para membros inferiores e superiores. Os testes de flexibilidade foram relativos à primeira e segunda avaliações. Posteriormente, os dados foram transportados para o programa SPSS WINDOWS 20, no qual foi realizada a estatística, pretendendo identificar e caracterizar relações entre as variáveis.

Para a análise dos dados, foram realizados testes de caráter descritivo para a identificação das médias e desvio padrão do pré e pós-teste. A seguir, foram realizados o teste " $\mathrm{t}$ " de Student, pareado, para verificar a possível diferença entre as médias do pré e pós-teste, o teste " $\mathrm{t}$ " de Student para amostras independentes (análise intragrupos) e ANOVA com Póst Hoc de Bonferroni para verificar a possível diferença entre as médias entre os grupamentos etários, ambos significativos para $\mathrm{p} \leq 0,05$. Para comparar as frequências observadas entre as categorias de classificação, foi utilizado o teste qui-quadrado de Pearson. 
Na Tabela 1, são apresentados os valores médios do desempenho das idosas no pré e pós-teste (avaliações 1 e 2), e na tabela 2, os resultados separados pelos grupamentos etários.

Tabela 1 - Valores médios do desempenho das idosas no pré-teste e pós-teste (avaliações 1 e 2).

\begin{tabular}{l|c|c|c|c|c}
\hline \multicolumn{1}{c|}{ Variável } & Avaliação & Média & DP & $\begin{array}{c}\mathbf{p} \\
\text { intragrupo }\end{array}$ & $\begin{array}{c}\mathbf{p ~ e n t r e} \\
\text { avaliações }\end{array}$ \\
\hline \multirow{2}{*}{$\begin{array}{l}\text { Flexibilidade } \\
\text { MS }\end{array}$} & 1 & $-8,38$ & 8,91 & $<0,001$ & \multirow{2}{*}{0,200} \\
\cline { 2 - 5 } & 2 & $-7,22$ & 9,03 & $<0,001$ & \\
\hline \multirow{2}{*}{$\begin{array}{l}\text { Flexibilidade } \\
\text { Ml }\end{array}$} & 1 & 2,71 & 9,09 & 0,041 & \multirow{2}{*}{0,002} \\
\cline { 2 - 5 } & 2 & 5,16 & 8,48 & $<0,001$ & \\
\hline
\end{tabular}

Ml: membros inferiores; MS: membros superiores.

Com base na Tabela 1, verifica-se que, tanto na flexibilidade de membros superiores como na de inferiores, houve melhoria dos valores médios do pré para o pós-teste, sendo a diferença de média superior nesta última variável. No entanto, em termos inferenciais, essa diferença só se mostra significativa em relação à flexibilidade de membros inferiores $(0,002)$. Nas análises intragrupos em cada variável e em cada uma das avaliações, constata-se grupos bem heterogêneos, haja vista que a única variável em que os resultados não diferem significativamente é a flexibilidade de membros inferiores, no pré-teste.

Tabela 2 - Diferenças entre as médias das diferentes avaliações das idosas, discriminadas por grupamento etário.

\begin{tabular}{|c|c|c|c|c|c|c|c|}
\hline Variável & Avaliação & $\begin{array}{l}60-64 \\
(n=15)\end{array}$ & $\begin{array}{l}65-69 \\
(n=15)\end{array}$ & $\begin{array}{l}70-74 \\
(n=8)\end{array}$ & $\begin{array}{c}75 \text { ou } \\
+ \\
(n=11)\end{array}$ & $\begin{array}{c}p \\
\text { valor }\end{array}$ & Post hoc* \\
\hline \multirow{2}{*}{$\begin{array}{l}\text { Flexibilidade } \\
\text { MS }\end{array}$} & 1 & $-4,02$ & $-11,58$ & $-2,37$ & $-14,73$ & $<0,001$ & $D<A, B, C$ \\
\hline & 2 & $-4,03$ & $-9,57$ & $-1,31$ & $-12,95$ & 0,012 & $D<A, C$ \\
\hline \multirow{2}{*}{$\begin{array}{l}\text { Flexibilidade } \\
\text { MI }\end{array}$} & 1 & 6,07 & 0,83 & 4,75 & $-1,14$ & 0,232 & - \\
\hline & 2 & 7,28 & 2,97 & 7,00 & 3,72 & 0,568 & - \\
\hline
\end{tabular}

*Bonferroni; A: 60-64 anos; B: 65-69 anos; C: 70-74 anos; D: 75 ou +. 
Na comparação do pré para o pós-teste apresentada na Tabela 2, observa-se que na variável membros superiores, o único grupamento em que não houve melhoria nos valores médios foi o de 60 a 64 anos, já entre os grupamentos em que foram observadas melhorias nos valores médios, a magnitude das diferenças apresentou-se, em ordem decrescente, nos grupamentos: 65 a 69 anos $(2,01), 75$ anos ou mais $(1,78)$ e 70 a 74 anos $(1,06)$. Na variável membros inferiores, todos os grupamentos obtiveram melhoras nos seus resultados, sendo que o grupo que obteve a maior melhora foi o grupamento de 75 anos ou mais $(4,86)$, seguido dos seguintes grupamentos etários: 70 a 74 anos (2.25), 65 a 69 anos $(2,14)$ e 60 a 64 anos $(1,21)$. Analisando a comparação entre os grupos etários, na variável flexibilidade de membros superiores, os resultados do teste inferencial evidenciam que o grupo mais idoso (75 anos ou mais) apresenta resultado significativamente inferior ao de todos os outros no pré-teste $(0,001)$, assim como aos grupamentos de 60 a 64 anos e de 70 a 74 anos $(0,012)$.

Tabela 3 - Categorização dos grupos de praticantes de ginástica funcional na flexibilidade de membros superiores e inferiores por meio dos critérios do protocolo de Rikli e Jones (2008).

\begin{tabular}{l|c|c|c|c|c|c|c}
\hline \multirow{2}{*}{ Variável } & \multicolumn{2}{|c|}{ Abaixo da média } & \multicolumn{2}{c|}{ Normal } & \multicolumn{2}{c|}{ Acima da média } & \multirow{2}{*}{$\begin{array}{c}\text { Valor } \\
\text { de } \mathbf{p}^{*}\end{array}$} \\
\cline { 2 - 7 } & $\mathbf{N}$ & $\%$ & $\mathbf{N}$ & $\%$ & $\mathbf{N}$ & $\%$ & \multirow{2}{*}{ \% } \\
\hline MS1 & 36 & 72,0 & 12 & 24,0 & 02 & 4,0 & $<001$ \\
\hline MS2 & 31 & 62,0 & 14 & 28,0 & 05 & 10,0 & 0,001 \\
\hline Ml1 & 29 & 58,0 & 13 & 26,0 & 08 & 16,0 & $<0,001$ \\
\hline Ml2 & 24 & 48,0 & 12 & 24,0 & 14 & 28,0 & 0,084 \\
\hline
\end{tabular}

Valor de $\mathrm{p}^{*}$ - calculado por meio do teste qui-quadrado de Pearson.

Com base nos resultados dos testes de flexibilidade apresentados pela Tabela 3, constata-se que, comparando o pré e pós-teste, na variável de membros superiores, ocorreu melhoria na classificação das idosas com uma redução de idosas categorizadas como abaixo da média $(10,0 \%)$ e consequente aumento nas categorias normal $(4,0 \%)$ e acima da média $(6,0 \%)$. Na variável flexibilidade de membros inferiores, percebe-se que houve um aumento considerável de idosas categorizadas como acima da média $(12,0 \%)$, embora tenha havido redução do número de idosas na categoria normal $(2,0 \%)$, ou seja, a melhoria se deu mais em função da redução do número de idosas na categoria abaixo da média $(10,0 \%)$. Convém salientar que, com exceção dos valores do IMC, nas variáveis flexibilidade de membros superiores e inferiores, foram observadas diferenças significativas nas duas avaliações. 
Membros superiores

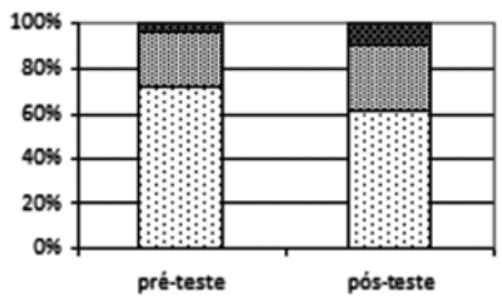

口-Média a Média $\mathbf{a}+$ Média
Membros inferiores

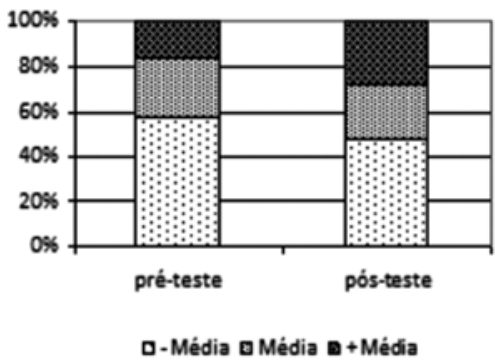

\section{Discussões}

O estudo comparou os resultados do pré e pós-teste para verificar se, após um período de treinamento de nove meses, é possível observar mudanças significativas na flexibilidade das idosas submetidas a um programa intervenção.

\subsection{Flexibilidade dos membros superiores}

A variável de membros superiores na analise do pré para o pós-teste não obteve resultados estatísticos significativos. Entretanto, quando se observa o comportamento do grupo nas categorias padronizadas do protocolo (abaixo da média, na média e acima da média), é possível identificar uma redução daquelas que apresentam médias abaixo do esperado. Esse resultado torna-se relevante na medida em que o programa de intervenção foi de ginástica funcional, que, mesmo apresentando-se como um programa constituído por um treinamento generalizado de atividade física, sem um foco específico na flexibilidade, é passível de contribuir, mesmo que essa contribuição seja a manutenção dos níveis de flexibilidade de membros superiores. Os resultados positivos obtidos por meio da ginástica funcional poderiam ser potencializados com a utilização de uma intervenção que enfatize a treinabilidade da flexibilidade, uma vez que se trata de uma valência tão relevante para a realização das tarefas cotidianas, principalmente em idosos. 
O estudo de Ribeiro et al. (2009) corrobora essa afirmação. O referido estudo avaliou 14 idosas participantes de um programa de ginástica com duração de 24 semanas. Na análise da flexibilidade, foi observado que, embora não se tenha observado diferença significativa entre as médias do pré e pós-teste, quando foi considerada a classificação, muitas passaram da categoria "regular" para a categoria "boa".

Outro estudo que teve como objetivo verificar a influência da flexibilidade na qualidade de vida e na autonomia de 69 idosas com média de idade de 65,33 anos, por meio de atividades de alongamento, observou que, semelhantemente ao presente estudo, houve melhora na flexibilidade com valores significativos no movimento de flexão horizontal do ombro e nos movimentos de abdução do ombro (VAREJÃO; DANTAS; MATSUDO, 2007). Ainda, Mayer e Lopes (2011) também observaram diferenças significativas nos resultados entre o pré e o pós-teste na variável de MMSS, avaliando 5 idosas com idade entre 61 e 73 anos, participantes de um projeto de Pilates solo, com exercícios semelhantes aos realizados pelas idosas do presente estudo, com 4 semanas de duração. Quando comparados os dados do pré e pós-teste, elas obtiveram uma melhora de 59,52\% no MMSS, sendo esta significativa.

Apesar de os estudos anteriormente mencionados especificarem a melhora da flexibilidade, verifica-se que, em sua maioria, trata-se de estudos em que o foco são experimentos cujo tratamento visou a melhoria da flexibilidade nessa população. Esse caráter reforça a relevância do presente estudo por ter observado melhora significativa na flexibilidade dos membros superiores mesmo quando o foco da intervenção não é a flexibilidade.

Observa-se que a flexibilidade dos 30 aos 70 anos sofre um declínio de 20 a 50\%, dependendo da articulação (HOLLAND et al., 2002). Esse aspecto ressalta a importância de um trabalho mais especifico para a flexibilidade entre os idosos, pois níveis adequados de flexibilidade e força, dentre outros, são determinantes para a execução de determinados movimentos, inclusive das atividades diárias. A redução da funcionalidade desses componentes pode acarretar numa grande dificuldade de realização dos movimentos que podem ser considerados obrigatórios para o cotidiano do idoso, acarretando em dependência do idoso e uma grande diminuição da sua qualidade de vida (HOLLAND et al., 2002; WARBURTON; GLEDHILL; QUINNEY, 2001). A diminuição da flexibilidade cria dificuldades de movimentação para os idosos, pois a redução da flexibilidade na extensão dos membros superiores pode causar muitos transtornos para o geronte, atrapalhando o uso das mãos e braços para a realização das suas atividades (BADLEY et al., 1984). 
Com base nos dados, na avaliação da treinabilidade, comparando as médias do pré e pós-teste, é possível verificar resultados significativos $(\mathrm{p}=0,002)$ no desempenho das idosas, evidenciando que uma intervenção de ginástica funcional é eficaz na melhoria da flexibilidade de membros inferiores.

O que se pode observar é que todas as médias de membros inferiores obtiveram melhora no pós-teste, porém, em termos inferenciais, nenhuma média obteve resultado significativo. Verifica-se também que a melhoria das médias foi maior entre as idosas com 75 anos ou mais, seguidas daquelas com 70 a 74 anos, 60 a 64 anos e 65 a 69 anos. Esses resultados sugerem que os dois grupos com mais idade foram mais favorecidos pelo programa de ginástica funcional. Tal resultado leva a supor que a melhoria mais acentuada pode estar relacionada a um nível inicial de flexibilidade mais deteriorado. Os resultados são classificados como "abaixo da média", "na média" e "acima da média", e conforme o protocolo utilizado pode-se considerar uma melhora muito expressiva da flexibilidade de membros inferiores entre o pré e o pós-teste, no qual obtivemos os resultados mais pertinentes, baixando em 10\% a categoria "abaixo da média", e em $2 \%$ a categoria "na média", aumentando, assim, o índice "acima da média" em $12 \%$ após os 9 meses de treinamento.

O presente estudo corrobora com a literatura ao constatar uma melhora expressiva da flexibilidade entre o pré e o pós-teste. Alves et al. (2004) analisaram a aptidão física relacionada à saúde de idosos com treinamento de hidroginástica ao longo de três meses. Foram acompanhadas 30 mulheres no grupo de estudo e 30 mulheres no grupo controle. Após ser submetido a aulas de hidroginástica duas vezes por semana, o grupo que praticou hidroginástica apresentou mudanças significativas na flexibilidade de membros inferiores.

Por outro lado, o estudo de Aguiar e Gurgel (2009) avaliou 26 mulheres com idade entre 60 e 80 anos, as quais foram divididas em dois grupos: o grupo de sedentárias ( $\mathrm{n}=13)$ e o grupo de praticantes de hidroginástica há pelo menos seis meses ( $n=13)$. O estudo teve como objetivo verificar a influência da hidroginástica sobre a qualidade de vida, a força de membros inferiores e a flexibilidade de idosas. Em se tratando da variável flexibilidade de membros inferiores, o grupo praticante de hidroginástica obteve resultados superiores ao grupo de sedentários após o período de intervenção proposto, o que corrobora com o nosso estudo, no qual o resultado do pós-teste foi superior ao do pré-teste.

Lima et al. (2010) avaliaram a influência de um programa de ginástica de dois meses de duração sobre a postura e a flexibilidade de 10 idosas 
institucionalizadas com idade entre 60 e 75 anos. Após o período de treinamento, foi verificado que algumas idosas $(40 \%)$ apresentaram progresso quanto à sua classificação de flexibilidade. Duas passaram da classificação "muita fraca" para a "fraca", uma para a classificação "alta", e outra passou a apresentar a classificação "muito alta". Assim como no presente estudo, o grupo que obteve os maiores ganhos de flexibilidade foi o grupo de idosas mais velhas. No presente estudo, o grupamento mais velho de 75 anos ou mais foi o que obteve os melhores resultados. Diferentemente do estudo de Hoefelmann et al. (2011), os autores verificaram que, com o aumento da idade, ocorre uma diminuição da distância alcançada.

O estudo de Vale et al. (2006) teve como objetivo verificar os efeitos do treinamento resistido na força máxima, na flexibilidade e na autonomia funcional de idosas. A amostra foi separada em dois grupos: o grupo força e o grupo controle. O grupo de força foi submetido a um treinamento contra resistência de força, por 16 semanas duas vezes na semana. Os resultados desse estudo corroboram com o do presente, pois foram obtidos resultados significativos do pré para o pós-teste no grupo de força na flexibilidade de membros inferiores (flexão de quadril, extensão do quadril, flexão do joelho).

Programas de atividade física para idosos podem diminuir em grande parte os efeitos do ciclo imobilidade (quedas, dor e medo), contribuindo para a manutenção ou ganho da flexibilidade e da força muscular, sendo esta uma meta muito importante em todos os programas de treinamento para idosos (DALEY; SPINKS, 2010). A redução da flexibilidade na extensão de joelho e flexão do quadril está relacionada com o declínio das atividades necessárias do idoso como deslocamento, curvar-se para alcançar algum objeto, dentre outros (LIMA et al., 2010). Com base nestes resultados, a prática regular de exercícios físicos tem sido recomendada como meio de diminuir ou reverter os efeitos negativos do envelhecimento (FIDELIS; PATRIZZI; WALSH, 2013). Uma boa amplitude articular proporciona condições para desenvolver atividades cotidianas ou, ainda, as mais complexas pelas quais os idosos podem ter a necessidade de desenvolver, tendo, assim, uma maior disposição para enfrentar os desafios do dia a dia (GONÇALVES; GURJÃO; GOBBI, 2007).

É importante destacar que não são encontrados, na literatura, estudos que se utilizam do método de ginástica funcional para idosas, assim como o presente. Dessa forma, as comparações realizadas se basearam naqueles estudos que trabalharam com outras atividades, mas ainda com o objetivo de desenvolver capacidades físicas semelhantes à ginástica funcional, como a flexibilidade. 
Observou-se, neste estudo, uma influência positiva do treinamento de ginástica funcional durante os nove meses de intervenção para a melhora dos níveis de flexibilidade das idosas de diferentes faixas etárias. Paradoxalmente, é o grupo mais idoso o mais favorecido pela intervenção por meio da ginástica funcional.

Os resultados do presente estudo evidenciam que as idosas podem se beneficiar com a melhoria ou manutenção da flexibilidade mesmo quando participam de um programa de intervenção que não tenha como foco único essa valência física. Sendo assim, sugere-se a manutenção e ampliação de propostas dessa natureza, procurando ampliar a clientela, bem como tentar sensibilizar idosos do sexo masculino a esse tipo de prática, haja vista que, em programas dessa natureza, a participação do sexo feminino é sempre maioritária. Por fim, considera-se relevante a realização de mais estudos que evidenciem o treinamento físico em idosas, principalmente da flexibilidade, que é de grande importância para a faixa etária em discussão.

\section{FLEXIBILITY IN ELDERLY FEMALES \\ PRACTITIONERS OF FUNCTIONAL EXERCISE}

abstract

We analyzed the flexibility in elderly practicing functional exercise. It was a causal comparative study within a sample of 50 elderly women. The range of tests was taken from the Rikli and Jones Methods. The average intragroups, between pre and posttest, age groups and the percentage of each category were compared. The statistical tests used were respectively: Student test "t" for independent sample, paired Student test "t", ANOVA and Qui square ( $p \leq 0.05)$. Significant results were found in lower limbs flexibility, and, between age groups in upper limbs flexibility. An intervention program of functional exercise contributes in improving and maintaining flexibility in elderly women.

keywords

Aging. Motor Activity. Elasticity. 
ACHOUR JUNIOR, Abdallah. Exercícios de alongamento: anatomia e fisiologia. 3 ed. São Paulo: Manole, 2009. 606 p.

AGUIAR, Jaina Bezerra de; GURGEL, Luilma Albuquerque. Investigação dos efeitos da hidroginástica sobre a qualidade de vida, a força de membros inferiores e a flexibilidade de idosas: um estudo no Serviço Social do Comércio - Fortaleza. Revista Brasileira de Educação Física e Esporte, São Paulo, v. 23, n. 4, p. 335-344, out./dez. 2009.

ALVES, Roseane Victor et al. Aptidão física relacionada à saúde de idosos: influência da hidroginástica. Revista Brasileira de Medicina do Esporte, São Paulo, v. 10, n. 1, p. 31-37, jan./fev. 2004.

BADLEY, Elizabeth; WAGSTAFF, Susan; WOOD, Philip. Measures of functional ability (disability) in arthritis in relation to impairment of range of joint movement. Annals of the Rheumatic Diseases, London, v. 43, n. 4, p. 563-569, Aug. 1984.

BENEDETTI, Maria Grazia et al. Effects of an adapted physical activity program in a group of elderly subjects with flexed posture: clinical and instrumental assessment. Journal of Neuroengineering and Rehabilitation, London, v. 5, p. 32, Nov. 2008.

BENEDETTI, Tânia Rosane Bertoldo; GONÇALVES, Lúcia Hisako Takase; MOTA, João Augusto Pinto da Silva. Uma proposta de política pública de atividade física para idosos. Texto \& Contexto Enfermagem, Florianópolis, v. 16, n. 3, p. 387-398, jul./set. 2007.

CARVALHO, Daniele Padilha; PETREÇA, Daniel Rogério. Adesão a prática de atividade física em indivíduos de 40-60 anos no município da Lapa - PR. Saúde e Meio Ambiente, Mafra, v. 1, n. 2, p. 53-65, dez. 2012.

CIPRIANI, Natália Cristina Santos et al. Aptidão funcional de idosas praticantes de atividades físicas. Revista Brasileira de Cineantropometria e Desempenho Humano, Florianópolis, v. 12, n. 2, p. 106-111, mar./abr. 2010.

DALEY, Monica J.; SPINKS, Warwick L. Exercise, mobility and aging. Sports Medicine, Auckland, v. 29, n. 1, p. 1-12, Jan. 2010.

FIDELIS, Luiza Teixeira; PATRIZZI, Lislei Jorge; WALSH, Isabel Aparecida Porcatti de. Influência da prática de exercícios físicos sobre a flexibilidade, força muscular manual e mobilidade funcional em idosos. Revista Brasileira de Geriatria e Gerontologia, Rio de Janeiro, v. 16, n. 1, p. 109-116, jan./mar. 2013.

FREITAS, Clara Maria Silvestre Monteiro de et al. Aspectos motivacionais que influenciam a adesão e manutenção de idosos a programas de exercícios física. Revista Brasileira de Cineantropometria e Desempenho Humano, Florianópolis, v. 9, n. 1, p. 92-100, mar. 2007.

GALLON, Daniela; GOMES, Anna Raquel Silveira. Idosos institucionalizados e os efeitos do exercício no processo de envelhecimento músculo esquelético: uma revisão. Revista Brasileira de Ciências do Envelhecimento Humano, Passo Fundo, v. 8, n. 1, p. 136-147, jan./abr. 2011.

GONÇALVES, Raquel; GURJÃO, André Luiz Demantova; GOBBI, Sebastião. Efeito de oito semanas de treinamento de força na flexibilidade de idosos. Revista Brasileira de Cineantropometria e Desempenho Humano, Florianópolis, v. 9, n. 2, p. 145-153, jun. 2007.

HOEFELMANN, Camila Peter et al. Aptidão funcional de mulheres idosas ativas com 80 anos ou mais. Motriz, Rio Claro, v. 17, n. 1, p. 19-25, jan./mar. 2011.

HOLLAND, George J. et al. Flexibility and physical functions of older adults: a review. Journal of Aging and Physical Activity, Champaign, IL, v. 10, n. 2, p. 169-206, Apr. 2002 
LIMA, Hélia Cristina Oliveira et al. Avaliação dos benefícios da ginástica localizada sobre a postura e a flexibilidade de mulheres na terceira idade. Revista Brasileira de Educação Física e Esporte, São Paulo, v. 24, n. 4, p. 525-534, out./dez. 2010.

MAYER, Arineti Priscila; LOPES, Wendell Arthur. A influência do método pilates na aptidão física de idosas do município de Guarapuava PR. VOOS: Revista Polidisciplinar Eletrônica da Faculdade Guairacá, Guarapuava, v. 3, n. 2, p. 82-92, dez. 2011.

MAZO, Giovana Zarpellon et al. Actividade física e qualidade de vida de mulheres idosas da cidade de Florianópolis, Brasil.Revista Portuguesa de Ciências do Desporto, Porto, v. 8, n. 3, p. 414-423, set./dez. 2008

$\mathrm{OH}$, Dong-hyun et al. Intensive exercise reduces the fear of additional falls in elderly people: findings from the Korea falls prevention study. Korean Journal of Internal Medicine, Seoul, v. 27, n. 4, p. 417-425, Dec. 2012.

RIBEIRO, Daniela Préve et al. Programa de ginástica para idosos nos centros de saúde: avaliação da aptidão funcional. Fisioterapia em Movimento, Curitiba, v. 22, n. 3, p. 407-417, jul./set. 2009.

RIKLI, Roberta E.; JONES, C. Jessie. Teste de aptidão física para idosos. Barueri: Manole, 2008.

SILVA, Margareth da; RABELO, Heloisa Thomaz. Estudo comparativo dos níveis de flexibilidade entre mulheres praticantes de atividade física e não praticantes. Movimentum: Revista Digital de Educação Física, Ipatinga, n. 1, v. 1, p. 1-15, ago./dez. 2006.

SILVA, Tamara Oliveira da et al. Avaliação da capacidade física e quedas em idosos ativos e sedentários da comunidade. Revista da Sociedade Brasileira de Clínica Médica, São Paulo, v. 8, n. 5, p. 392-398, set./out. 2010.

VALE, Rodrigo Gomes de Souza et al. Efeitos do treinamento resistido na força máxima, na flexibilidade e na autonomia funcional de mulheres idosas. Revista Brasileira de Cineantropometria e Desempenho Humano, Florianópolis, v. 8, n. 4, p. 52-58, dez. 2006.

VAREJÃO, Ronaldo Vivone; DANTAS, Estélio Henrique Martins; MATSUDO, Sandra Marcela Mahecha. Comparação dos efeitos do alongamento e do flexionamento, ambos passivos, sobre os níveis de flexibilidade, capacidade funcional e qualidade de vida do idoso. Revista Brasileira de Ciência e Movimento, São Paulo, v. 15, n. 2, p. 87-95, 2007.

VERAS, Renato. Envelhecimento populacional contemporâneo: demandas, desafios e inovações. Revista de Saúde Pública, São Paulo, v. 43, n. 3, p. 548-554, maio/jun. 2009.

VIRTUOSO JÚNIOR, Jair Sindra; GUERRA, Ricardo Oliveira. Fatores associados às limitações funcionais em idosas de baixa renda. Revista da Associação Médica Brasileira, São Paulo, v. 54, n. 5, p. 430-435, set./out. 2008.

WARBURTON, Daren E. R.; GLEDHILL, Norman; QUINNEY, Arthur. The effects of changes in musculoskeletal fitness on health. Canadian Journal of Applied Physiology, Champaign, IL, v. 26, n. 2, p. 161-216, Apr. 2001. 